\title{
Long-Term Outcomes of Patients with Stent Tips Embedded into Internal Carotid Artery Branches during Aneurysm Coiling
}

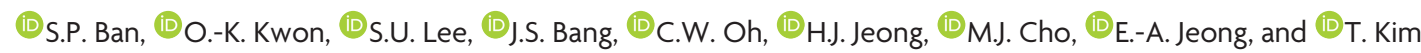

\begin{abstract}
BACKGROUND AND PURPOSE: During stent-assisted coiling of ICA aneurysms, stent tips are sometimes unintentionally embedded into ICA branches. Stent tips can be visualized because they have radiopaque markers. Concerns regarding stent tip misplacement include risks of artery perforation and occlusion. The aim of this study was to evaluate the long-term outcomes of ICA branches with embedded stent tips.
\end{abstract}

MATERIALS AND METHODS: ICA branches with embedded stent tips were identified among 35 patients with unruptured ICA aneurysms treated with stent-assisted coiling between November 2003 and November 2014. Patient clinical and angiographic outcomes associated with the embedded stent tip were analyzed.

RESULTS: Most of the 35 studied aneurysms were paraclinoid ICA aneurysms $(n=30)$. The most commonly involved ICA branch was the posterior communicating artery (26 patients, $74.3 \%$ ), followed by the anterior choroidal artery (8 patients, $22.9 \%$ ) and ophthalmic artery ( 1 patient, 2.9\%). During the follow-up period (38.6 \pm 17.9 months), no new neurologic deficits developed. Neither hemorrhagic nor thromboembolic events occurred. Angiography was performed during the final follow-up evaluation at a mean of $32.7 \pm 18.0$ months, and all ICA branches with embedded stent tips showed patent blood flow without severe luminal narrowing.

CONCLUSIONS: In our experience, placement of a stent tip into ICA branches during stent-assisted coiling was not associated with any major adverse events.

ABBREVIATIONS: AchoA = anterior choroidal artery; OphA = ophthalmic artery; PcomA = posterior communicating artery; SAC = stent-assisted coiling

S elf-expandable stents have provided considerable assistance in increasing the indications for aneurysm coiling. Complex and wide-neck aneurysms, which are considered challenging or impossible to treat with simple coiling, can now be treated. ${ }^{1}$

Generally, self-expandable stents for cerebral aneurysm treatment have proximal and distal radiopaque markers to identify the end of the stent. These markers are made from coiled tantalum wire or platinum bands, which are larger than the stent strut itself. The markers are attached to the triangular pointed stent tip wire (strut) part. During stent-assisted coiling (SAC), the stent tip can sometimes be unintentionally em-

Received November 7, 2017; accepted after revision January 3, 2018

From the Department of Neurosurgery, Seoul National University Bundang Hospital, Seongnam, Gyeonggi-do, Korea.

Please address correspondence to Tackeun Kim, MD, Department of Neurosurgery, Seoul National University Bundang Hospital, 82 Gumi-ro 173 beon-gil, Bundang-gu, Seongnam-si, Gyeonggi-do, 13620, Korea; e-mail: midabo@naver.com

三 Indicates article with supplemental on-line table.

http://dx.doi.org/10.3174/ajnr.A5583 bedded into a small arterial branch. We have occasionally encountered this event, particularly during SAC performed for ICA aneurysms, which is well-visualized due to the radiopaque markers. This phenomenon raises concerns regarding the potential risks of arterial perforation due to the sharp end and constant arterial pulsating motion of the stent, as well as the chances of vessel occlusion. Stent tip markers may also disrupt blood flow by themselves or by intimal injury (thrombus formation or intimal hyperplasia), which may then lead to infarction. Despite these concerns, we were unable to find a report regarding the clinical or radiologic outcomes of embedded stent tips within ICA branches. Thus, we reviewed our series of patients who had undergone SAC for unruptured ICA aneurysms and in whom stent tips were embedded within the ICA branch orifice. We analyzed clinical and angiographic data to identify the outcomes of these cases.

\section{MATERIALS AND METHODS \\ Patients}

This study was approved by the institutional review board at our institution. Informed consent from enrolled patients was waived. 
Coil embolizations in author's institution between

November, 2003 and November, 2014 (2998 aneurysms in 2730 patients)

Unruptured intracranial aneurysms treated with stent-assisted coiling

(947 aneurysms in 886 patients)

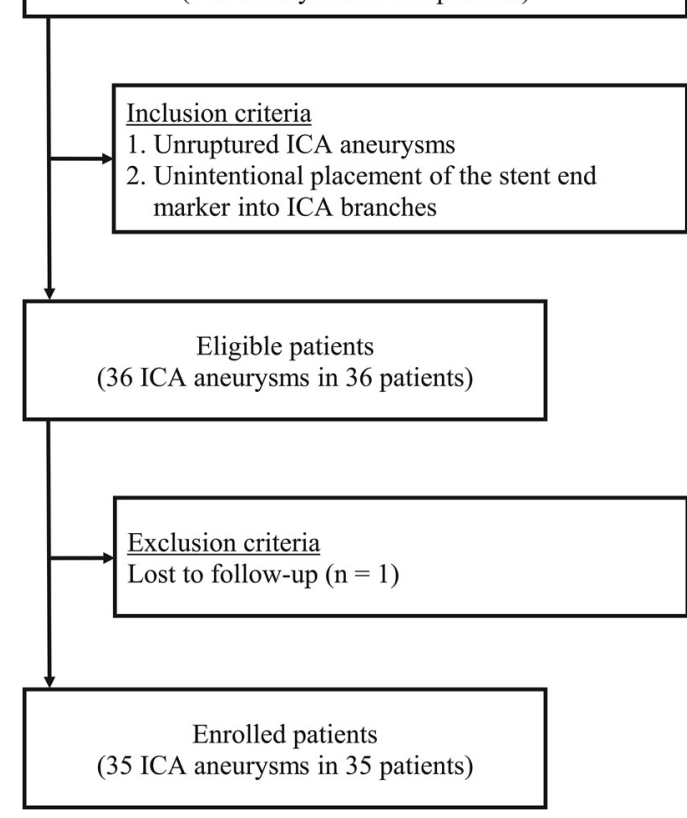

FIG 1. Outline of patient selection.

We retrospectively reviewed patient medical records, including pre- and posttreatment radiologic studies. Our data base contained a series of 2730 patients with 2998 intracranial aneurysms treated with endovascular techniques between November 2003 and November 2014. Among these patients, 886 with 947 unruptured intracranial aneurysms were treated with SAC. In this series, data from 664 patients with 706 ICA aneurysms who were treated with SAC were collected and reviewed. Among these patients, the placement of a stent tip into an ICA branch was identified in 36 aneurysms of 36 patients. In our subsequent analysis, 1 patient who was lost to follow-up was excluded. The patient selection criteria are outlined in Fig 1.

\section{Antiplatelet Therapy Protocol and Endovascular Procedures}

Patients with unruptured aneurysms received dual antiplatelet agents (100 mg of aspirin and $75 \mathrm{mg}$ of clopidogrel) for at least 5 days before embolization. One day before coil embolization, P2Y12 reaction units were measured using VerifyNow (Accumetrics, San Diego, California). Patients with clopidogrel resistance (>220 P2Y12 reaction units) received a modified antiplatelet regimen. Details regarding our antiplatelet agent protocol have been previously described. ${ }^{2}$ A 3000-IU bolus dose of intravenous heparin was administered after the placement of the femoral artery sheath, and heparin was later infused at an hourly booster dose of $1000 \mathrm{IU}$, with monitoring of the activated clotting time. After the procedure, dual antiplatelet treatment continued for 1 year; after
1 year, this therapy was exchanged for daily oral treatment with $100 \mathrm{mg}$ of aspirin for an additional year.

All endovascular procedures were performed with the patient under general anesthesia using an Integris Allura scanner (Philips Healthcare, Best, the Netherlands) before 2014 and an IFNX$8000 \mathrm{~V}$ scanner (Toshiba, Tokyo, Japan) from 2014. The jailing technique was mainly used for SAC. First, a target aneurysm was selected using a microcatheter (Excelsior SL-10; Stryker Neurovascular, Kalamazoo, Michigan). Then, the parent artery was navigated with another microcatheter (Prowler Select Plus, Codman \& Shurtleff, Raynham, Massachusetts; or Neuro Renegade, Stryker Neurovascular) for stent delivery. A stent was deployed into the parent artery across the aneurysm neck. Then, the aneurysm was coiled using platinum detachable coils until complete occlusion was achieved or further coiling was deemed unsafe. Stent type, length, and placement were determined by the neurovascular team and were based on aneurysm location and neck diameter. Among 706 ICA aneurysms treated with SAC, Enterprise stents (Codman \& Shurtleff) were most commonly used ( $n=607,86.0 \%$ ), followed by Neuroform stents (Stryker Neurovascular) in 71 cases $(10.1 \%)$. The other stents used included Low-Profile Visualized Intraluminal Support stents (MicroVention, Tustin, California) in 19 cases $(2.7 \%)$ and Solitaire AB stents (Covidien, Irvine, California) in 9 cases $(1.3 \%)$. Of the 35 cases, 32 were treated with a single Enterprise stent and 3 were treated with a single Neuroform stent.

\section{Stent Tips of Enterprise and Neuroform Stents}

The Enterprise stent is a laser-cut stent with a closed-cell design. This stent has flared proximal and distal ends, consisting of 4 pointed parts. Each end has 4 radiopaque tantalum coil markers for increased visibility, which flare out for fixation of the stent onto the vascular wall when fully deployed (Fig $2 A$ ). Due to the sharp, pointed, flaring parts, these stents can be embedded into the small ICA branches. The radiopaque markers of the tips allow them to be clearly visualized. The diameter of a radiopaque marker was thicker than that of the stent strut. Stent strut width and thickness were approximately 0.0015 inches $(0.0381$ $\mathrm{mm})$ and 0.0031 inches $(0.0787 \mathrm{~mm})$, respectively. The thickness and length of the radiopaque marker were approximately 0.008 inches $(0.2032 \mathrm{~mm})$ and 0.043 inches $(1.0922 \mathrm{~mm})$, respectively (Fig $2 B,-C$ ).

The Neuroform stent is a laser-cut stent with an open-cell or half-open-cell design. This stent has 4 distal and 4 proximal radiopaque platinum markers (Fig 2D). Each open-cell segment may serve as a separate fixing device to enhance the apposition of the stent to the vessel wall like the flared ends of the Enterprise stent. Like the Enterprise stent, the stent tip may be placed and embedded into the branch orifice. Stent strut width and thickness were approximately 0.0027 inches $(0.0686 \mathrm{~mm})$. The thickness and length of the radiopaque marker were approximately 0.0116 inches $(0.2946 \mathrm{~mm})$ and 0.030 inches $(0.762 \mathrm{~mm})$, respectively (Fig 2E, $-F$ ).

\section{Evaluation of Clinical and Radiologic Outcomes}

The clinical outcomes of all patients were evaluated using the mRS score on admission and at the final outpatient follow-up visit. 

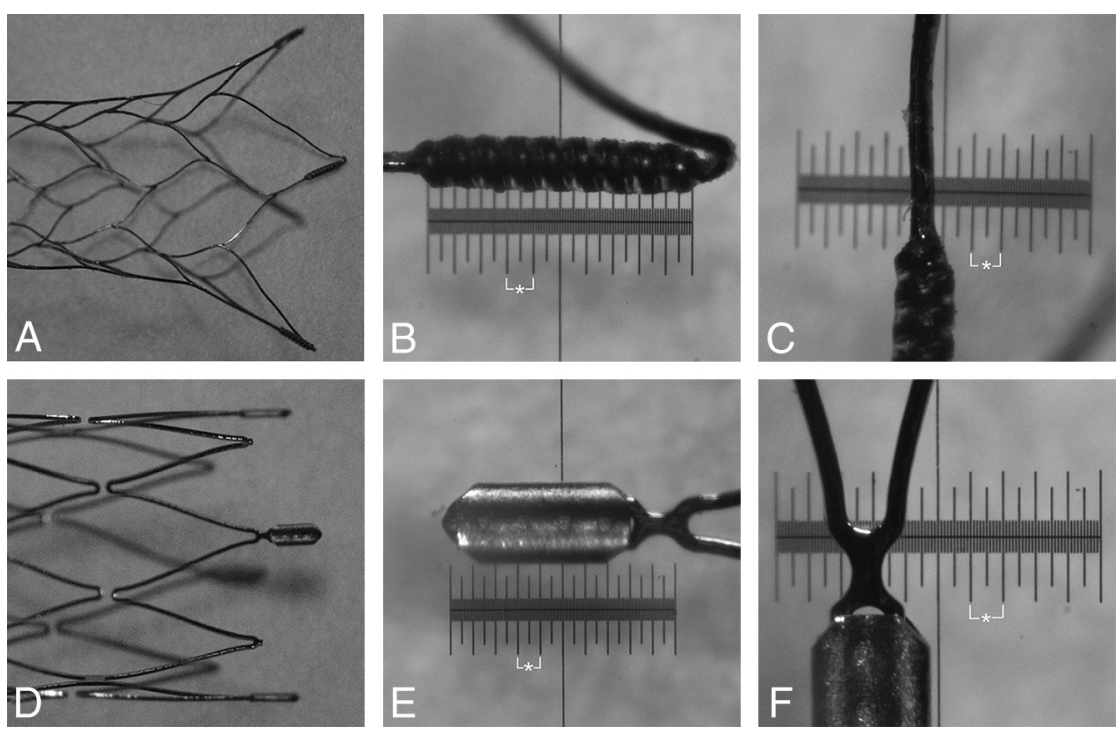

FIG 2. A, Flared ends of the Enterprise stent. $B$, Length of the flare marker of the Enterprise stent. $C$, Width of the flare marker and stent strut of the Enterprise stent. $D$, End of the Neuroform stent. $E$, Length of the platinum marker of the Neuroform stent. F, Width of the platinum marker and stent strut of the Neuroform stent. Asterisk indicates $0.1 \mathrm{~mm}$.

Newly developed neurologic deficits as well as hemorrhagic and thromboembolic complications during follow-up periods were also reviewed. The placement and embedding of a stent tip with a marker into a branching artery was defined as a stent tip that was placed at the level of branching arteries, with the tip marker clearly visualized within the artery orifice via angiographic views, including rotational angiograms. According to our institutional protocol, patients underwent imaging follow-up at 3, 6, 12, 24, and 36 months with skull plain radiography and at 1,2 , and 3 years with 3D-TOF MRA, including source imaging. Any time a major recanalization was found, DSA was performed. DSA follow-up studies were routinely conducted between 3 and 5 years after coil embolization if no major recanalization of an aneurysm was suspected. In this study, each patient underwent a minimum of 12 months of radiologic follow-up. Vessel patency was documented by follow-up DSA. If follow-up DSA was not performed, vessel patency was confirmed using the follow-up axial MRA source images.

\section{Data Analysis}

All statistical analyses were performed using SPSS software (Version 22.0; IBM, Armonk, New York). Continuous variables are presented as mean $\pm \mathrm{SD}$. Binary variables were compared using the Fisher exact test. Statistical significance was set at $P<.05$.

\section{RESULTS}

The overview of patients with stent tips embedded into the ICA branches is summarized in the On-line Table. Eight (22.9\%) patients were men, and $27(77.1 \%)$ were women. The mean age was $49.3 \pm 12.8$ years (range, 21-76 years). The cases included 30 (85.7\%) paraclinoid ICA aneurysms, $3(8.6 \%)$ ophthalmic artery (OphA) aneurysms, 1 (2.9\%) cavernous ICA aneurysm, and 1 $(2.9 \%)$ posterior communicating artery (PcomA) aneurysm. The most common ICA branching artery with an embedded stent tip was the PcomA (26 patients, 74.3\%). Other involved branching arteries included the anterior choroidal artery (AchoA) in $8(22.9 \%)$ patients and the OphA in $1(2.9 \%)$ patient. The incidence of stent tips embedded within ICA branches was not associated with the type of stent (closed-cell-design stent, $5.3 \%$, versus open-cell-design stent, $4.2 \% ; P=.424)$. Most of these events occurred during deployment of the distal end of the stent at the upward ICA curvature near the ICA branches (32 cases, $91.4 \%$ ), followed by unintentional stent advancement during microcatheter manipulation ( 3 cases, $8.6 \%$ ).

The mean clinical follow-up duration was $38.6 \pm 17.9$ months (range, 12-91 months). The preoperative $\mathrm{mRS}$ score was 0 in $30(85.7 \%)$ patients and 1 in $5(14.3 \%)$ patients. The final follow-up mRS score was 0 in 32 (91.4\%) patients and 1 in $3(8.6 \%)$ patients. No patient experienced mRS score deterioration. No newly developed neurologic deficits were observed. Neither hemorrhagic nor thromboembolic (or ischemic) complications occurred during the follow-up period.

The mean angiographic follow-up duration was $32.7 \pm 18.0$ months (range, 12-91 months). The mean initial diameter of the ICA branches with embedded stent tips was $1.79 \pm 0.69 \mathrm{~mm}$ (range, 0.51-2.81 mm). On final follow-up angiography (15 cases were measured by MRA with source imaging and 20 cases were measured by DSA), all ICA branches with an embedded stent tip had persistent blood flow without severe luminal narrowing. In 1 case, the radiopaque stent marker appeared to penetrate the PcomA. In the angiogram, the marker appeared to be located outside the arterial wall. However, no clinical event was observed (case 11).

\section{Illustrative Cases}

Case 11. A 64-year-old patient presented with an unruptured left paraclinoid ICA aneurysm (Fig $3 A,-B$ ). The aneurysm was treated with coil embolization using a $4.5 \times 20 \mathrm{~mm}$ Neuroform stent. Postembolization angiography showed the placement of the stent marker in the PcomA (Fig 3C, $-D$ ). Although a radiopaque stent marker appeared to penetrate the PcomA in the 5-year-follow-up angiograms, the PcomA was patent and no hemorrhagic complication occurred (Fig 3E, $-F$ ).

Case 13. A 23-year-old patient presented with an unruptured left paraclinoid ICA aneurysm (Fig 4A, -B). The aneurysm was treated with coil embolization using a $4.5 \times 22 \mathrm{~mm}$ Enterprise stent. Postembolization angiography showed the placement of the stent marker into the AchoA (Fig 4C, - D). Follow-up angiography at 3 years showed patent blood flow to the AchoA without severe luminal narrowing (Fig $4 E,-F)$. 

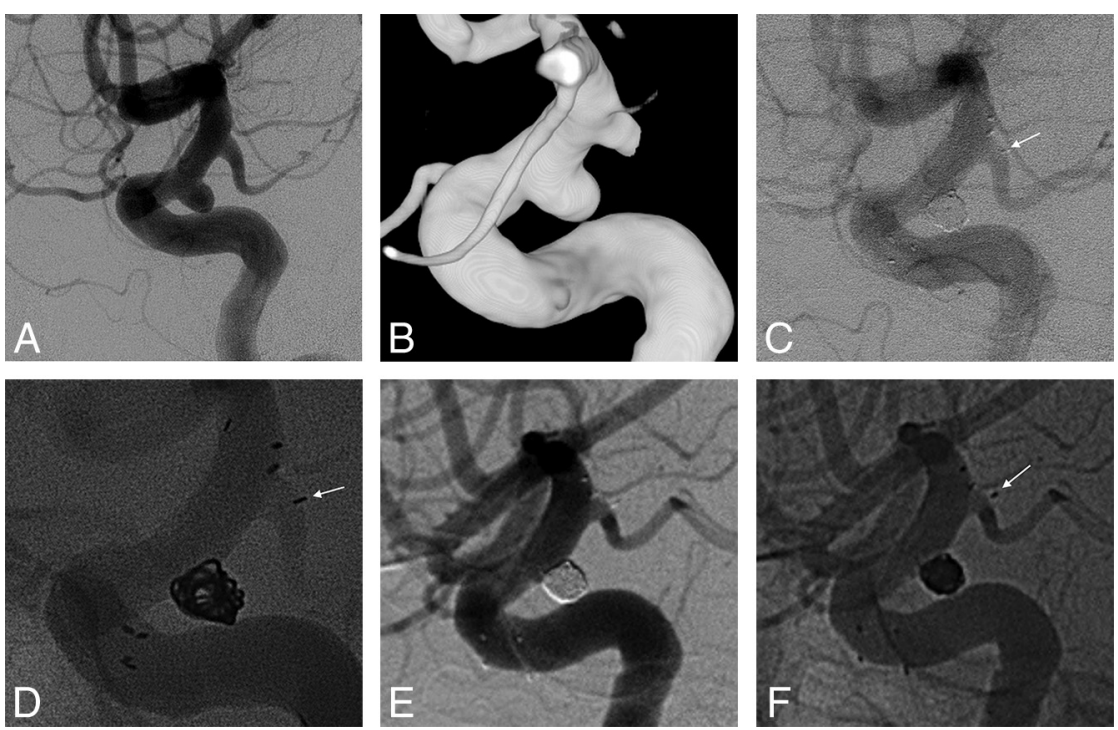

FIG 3. A, An unruptured left paraclinoid ICA aneurysm on pre-embolization DSA. $B, 3 D$ image of the aneurysm. $C$ and $D$, A distal stent marker of the Neuroform stent (arrow) is deployed into the posterior communicating artery, as shown on postembolization angiography. $E$ and $F$, The blood flow to the PcomA with malposition of the stent marker is patent on 5-year-follow-up cerebral angiography. A radiopaque stent marker appears to penetrate the PcomA (arrow).
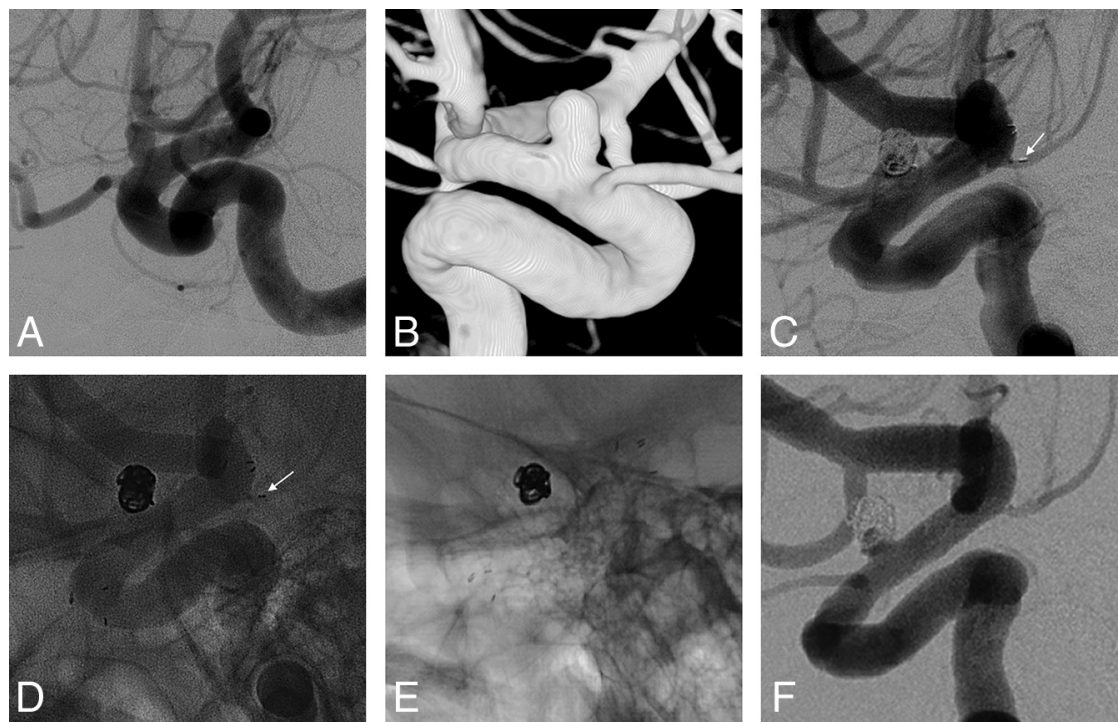

FIG 4. A left paraclinoid ICA aneurysm is shown on pre-embolization DSA $(A)$ and $3 D$ imaging $(B)$. $C$ and $D$, Postembolization angiography shows the malposition of the Enterprise stent marker in the anterior choroidal artery (arrow). $E$ and $F$, Follow-up cerebral angiography at 3 years shows persistent blood flow to the AchoA.

\section{DISCUSSION}

This study demonstrates that stent tip embedding into the ICA branches during SAC of cerebral aneurysms was not associated with any major adverse events. No arterial rupture or occlusion was observed during at least 1 year (12-91 months) of follow-up.

During SAC of ICA aneurysms, stent tips can be deployed and unintentionally embedded into ICA branching arteries due to their characteristics. In our study, the incidence of this event was $5.1 \%$, and the PcomA was the most commonly involved ICA branch, followed by the AchoA. Although we attempted to deploy stents so as avoid embedding the stent tip into the orifice of these
ICA branches, the distal part of a stent may be deployed flat around the PcomA and AchoA due to the ICA curvature, resulting in stent tips embedding into these ICA branches. In this study, this issue was the most common cause of an embedded stent tip within ICA branches (91.4\%). Because the ICA usually starts to curve upward near the PcomA and the diameter of the PcomA is commonly larger than that of the AchoA, it is likely that the stent tips are more easily embedded into the PcomA than into the AchoA.

Regarding the structure of the stents, because a closed-cell-design stent immediately transmits a force from one end to the other end, embedding of a stent tip into ICA branches due to stent advancement during microcatheter manipulation may be more commonly observed in patients receiving a closed-cell-design stent. Concerning this possible mechanism, in this study, all 3 cases $(8.6 \%)$ in which the distal part of a stent was initially deployed proximally near the PcomA and then advanced and embedded into the PcomA due to microcatheter manipulation were treated with the Enterprise stent (closedcell design). However, because the most common cause of embedding of a stent tip into ICA branches was the ICA curvature, no association was observed among the types of stents used in this study.

This event raises the following concerns: vessel rupture (perforation) and occlusion. Vessel rupture due to the forceful advancement of stents has been reported. The sharp ends of the stent may contribute to arterial rupture., The lack of this complication in our study suggests that stent ends were inserted into the branching arteries without excessive force. The presence of an embedded stent tip in small arteries can be a risk factor for further arterial injury with persistent arterial pulsation, at least theoretically. However, this study shows that this risk is not likely in practice.

Vessel occlusion or significant flow disruption may develop through intimal injury by the stent tip in a delayed manner as well as mechanical occlusion by stent tips and large markers themselves during the acute period. Therefore, we can consider several plausible mechanisms of vessel occlusion or flow disruption. A stent covering the small arterial ostium does not usually lead to arterial occlusion. ${ }^{5-7}$ Unlike a simple stent covering the arterial 
orifice, embedded stent tips have greater luminal occupancy due to the sharp triangular stent tip labeled with a radiopaque marker. Radiopaque markers at the stent tip have a larger area and volume than the stent. In a coronary artery study of a swine model, 2-fold thicker stents were $49 \%$ more thrombogenic and increased flow stagnation and disruption. ${ }^{8}$ Stent markers are 5.3-fold thicker than the stent strut in the Enterprise stent and 4.3-fold thicker than the stent strut in the Neuroform stent. In addition, persistent arterial pulsation with stent tips at the arterial orifice may also lead to further repeat intimal injury and subsequent thrombus formation and neointimal hyperplasia. ${ }^{4,9}$ However, this issue was not detected in our series of patients. The triangular shape of the stent tip may reduce such risks by stopping further advancement of the stent strut into the arterial lumen. The persistent need for blood flow to the ICA branches may also help prevent occlusion or severe stenosis. $^{10}$

Furthermore, luminal narrowing by intimal hyperplasia may be spontaneously reversed. ${ }^{11}$ The proliferated neointima reaches a maximal thickness by 2 months, and this neointimal layer gradually becomes thin, more sclerotic, and less cellular by 8 months. Kim et $\mathrm{al}^{9}$ reported that most in-stent stenoses would spontaneously improve to $91 \%$ of the initial mean diameter after 24 months. In our series, we did not perform angiographic follow-up until 12 months after SAC. Thus, luminal narrowing before this time was not confirmed. However, neither ischemic symptoms nor infarction developed in our study. One case (case 11) showed a radiopaque stent marker that appeared to be outside the PcomA lumen on 5-year-follow-up angiographic images. It is uncertain whether the stent tip was located outside the artery. However, no clinical symptoms or radiologic findings associated with arterial rupture were observed immediately after coiling, which suggests that the marker was more likely inside the artery and encased by neointimal hyperplasia. Although the mechanism has not yet been clearly determined, this study demonstrates that vessel occlusion by an embedded stent tip into the lumen is not common in practice.

Our study has several limitations including its retrospective nature and small sample size from a single institution. Furthermore, this study showed only the results of placement of stent markers from Enterprise and Neuroform stents, which are only 2 varieties among many expandable stents. In 15 cases, follow-up imaging was performed with TOF-MRA according to our institutional protocol. Although the patency of ICA branches with embedded stent tips was confirmed by MRA images, we did not show the changes in the diameters of ICA branches with embedded stent tips because this would be inaccurate due to the metal artifacts of the embedded stent tip on MRA images. Further research using computational fluid dynamics is warranted to more completely understand the changes in the diameter of ICA branches with embedded stent tips and the difference in the flow disturbance effect between stent struts and radiopaque markers.

\section{CONCLUSIONS}

In our experience, placement of a stent tip into ICA branches during SAC was not associated with any major adverse events. During 12-91 months of follow-up, vessel rupture, significant blood flow reduction, and arterial occlusion were not detected.

\section{REFERENCES}

1. Geyik S, Yavus K, Yurttutan N, et al. Stent-assisted coiling in endovascular treatment of $\mathbf{5 0 0}$ consecutive cerebral aneurysms with long-term follow-up. AJNR Am J Neuroradiol 2013;34:2157-62 CrossRef Medline

2. Hwang G, Huh W, Lee JS, et al. Standard vs modified antiplatelet preparation for preventing thromboembolic events in patients with high on-treatment platelet reactivity undergoing coil embolization for an unruptured intracranial aneurysm: a randomized clinical trial. JAMA Neurol 2015;72:764-72 CrossRef Medline

3. Chalouhi N, Jabbour P, Singhal S, et al. Stent-assisted coiling of intracranial aneurysms: predictors of complications, recanalization, and outcome in $\mathbf{5 0 8}$ cases. Stroke 2013;44:1348-53 CrossRef Medline

4. Wang CC, Li W, Feng ZZ, et al. Preliminary experience with stent-assisted coiling of aneurysms arising from small $(<2.5$ $\mathrm{mm}$ ) cerebral vessels using the Low-Profile Visualized Intraluminal Support Device. AJNR Am J Neuroradiol 2017;38:1163-68 CrossRef Medline

5. D'Urso PI, Lanzino G, Cloft $\mathrm{HJ}$, et al. Flow diversion for intracranial aneurysms: a review. Stroke 2011;42:2363-68 CrossRef Medline

6. Masuo O, Terada T, Walker G, et al. Study of the patency of small arterial branches after stent placement with an experimental in vivo model. AJNR Am J Neuroradiol 2002;23:706-10 Medline

7. Seong J, Wakhloo AK, Lieber BB. In vitro evaluation of flow divertors in an elastase-induced saccular aneurysm model in rabbit. J Biomech Eng 2007;129:863-72 CrossRef Medline

8. Kolandaivelu K, Swaminathan R, Gibson WJ, et al. Stent thrombogenicity early in high-risk interventional settings is driven by stent design and deployment and protected by polymer-drug coatings. Circulation 2011;123:1400-09 CrossRef Medline

9. Kim YS, Lee SW, Yeom JA, et al. Angiographic findings of in-stent intimal hyperplasia after stent-assisted coil embolization: are they permanent findings? J Neurosurg 2016;124:328-33 CrossRef Medline

10. Iosif C, Berg P, Ponsonnard S, et al. Role of terminal and anastomotic circulation in the patency of arteries jailed by flow-diverting stents: animal flow model evaluation and preliminary results. J Neurosurg 2016;125:898-908 CrossRef Medline

11. Schatz RA, Palmaz JC, Tio FO, et al. Balloon-expandable intracoronary stents in the adult dog. Circulation 1987;76:450-57 CrossRef Medline 